\title{
Juri Toomre and the art of modeling convection zones
}

\author{
Jean-Paul Zahn \\ LUTH, Observatoire de Paris, CNRS UMR 8102, Université Paris Diderot \\ 5 place Jules Janssen, 92195 Meudon, France \\ email: Jean-Paul.Zahn@obspm.fr
}

\begin{abstract}
Thermal convection plays a very important role in the structure and evolution of stars, as it is one of the main physical processes that transport heat from their interior where it is released, to the surface where it is radiated into space. Much progress has been achieved in modeling that process during the past 60 years, and I shall recall here how Juri Toomre has greatly contributed to it.
\end{abstract}

Keywords. convection; turbulence; stars: interiors.

\section{Early work on stellar convection}

It all began with the modeling of fully convective stars which, to a good approximation, are adiabatically stratified and are therefore polytropes of degree $3 / 2$. If all stars were such polytropes, stellar astrophysics would be a very simple matter, captured by a differential equation of second order that we owe to Lane and Emden. But thanks to Eddington (1926), the pendulum swung to the other side, although his opinion was a bit extreme. I cannot resist quoting him: "We shall not enter further into the historic problem of convective equilibrium since modern researches show that the hypothesis is untenable. In stellar conditions the main process of transport of heat is by radiation and other modes of transfer may be neglected."

As often, the truth was lying in between. Unsöld (1930) pointed out that, due to the ionization of hydrogen, the solar atmosphere would be unstable just below the surface, according to the Schwarzschild criterion; and indeed, solar granulation was clearly the signature of convective motions, as it had been already suggested by Emden. Soon solar models were built with a convective envelope and a stable radiative interior (cf. Cowling 1936). To treat that convection, and to describe the transition from radiative to convective transport of heat, Ludwig Biermann (1938) borrowed from Prandtl the concept of mixinglength - the mean free path of convective eddies, much as that of molecules in the kinetic theory of gas. This concept was later applied by Erika Böhm-Vitense to produce the first realistic model of the Sun (Vitense 1953); she chose to scale the mixing-length $\ell$ with the pressure scale-height $H_{P}$, and she showed that the depth of the solar convection zone depends sensitively on the value of the mixing-length parameter $\alpha=\ell / H_{P}$.

Since that parameter cannot be derived from first principles, it must be calibrated by adjusting evolutionary models to the Sun, after they have been run to solar age. But it is still a matter of debate how this calibration should be extended to stars of different mass and other evolutionary states.

The shortcomings of the mixing-length treatment are well known; beyond the uncertainties afflicting the mixing-length parameter, there is the fact that the convective flux is a local function of the superadiabatic gradient, which does to not allow for penetration or overshoot. In spite of its deficiencies, MLT is still widely used, because it is so simple to 


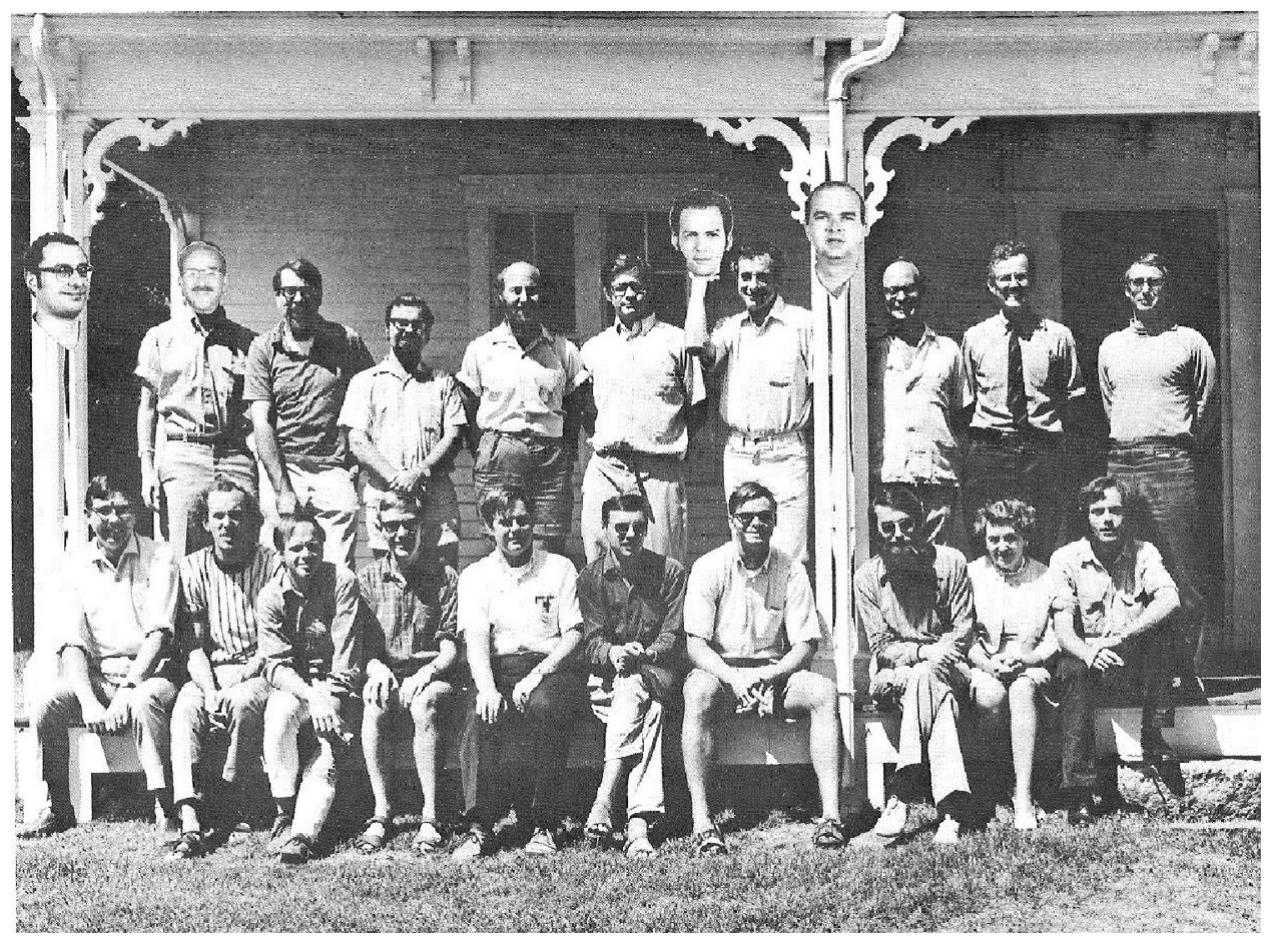

Figure 1. Woods Hole Summer School 1969. Standing (left to right): Buschi (posted), Stern, Prendergast, Gough, Keller, Kraichnan, Spiegel (being supported by) Veronis, Toomre (pillared), Harrison, Backus, Malkus. Seated: Zahn, Defouw, Gans, McKee, Trasco, Perdang, Barker, Auré, Thayer, Mészáros.

implement in a stellar evolution code. Valuable attempts have been made to improve the prescription by introducing higher order correlations, notably by Da-Run Xiong (1985) and Vittorio Canuto (1992), but these involve several additional parameters which - like $\alpha$ - cannot be derived from first principles. For most purposes that go beyond standard stellar evolution, a more realistic description of convection is required, and Juri Toomre has dedicated most of his scientific carreer to accomplish this task.

\section{A step forward : the modal treatment}

I met Juri in July 1969, on Cape Cod, where we were participating in a summer school with Douglas Gough, at the Woods Hole Oceanographic Institution. This school was founded in 1959 by Willem Malkus, George Veronis, Ed Spiegel and a few others, "with the aim of introducing a then relatively new topic in mathematical physics, geophysical fluid dynamics, to graduate students in physical sciences", as one can read on its website. The school is attended by a dozen or so graduate students, and as many senior researchers. It lasts ten weeks: two weeks of lectures, and thereafter the students work on a project under the guidance of a senior participant. Once in a while, the subject of the school is on astrophysics, as it was the case in 1969. Figure 1 shows the participants of that session; Ed and Juri were absent when the picture was taken - admire how they have been included nevertheless!

It was there in Woods Hole that I learned from the work undertaken by Ed with Douglas and Juri, to give a more physical representation of thermal convection, rooted 


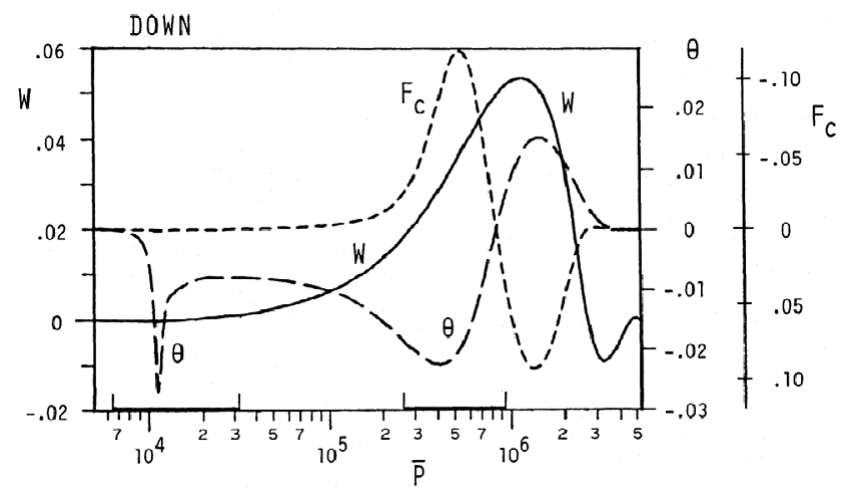

Figure 2. The modal treatment applied to convection in an A-type star. Variation with depth (or mean pressure $\bar{P}$ ) of the amplitude functions for the vertical momentum density $W$ and the relative variation $\Theta$ of temperature; also displayed is the convective heat flux $F_{C}$. The horizontal plane is tiled with hexagonal cells in which the flow is predominantly downwards $(W>0)$ at cell center. Such convection of large horizontal scale is buoyancy driven in the second unstable zone and penetrates with vigor into the stable region below, resulting in countercells at greater depth. The motions also extend upward through the interzone and establish a link between the two unstable zones: the entire domain is thoroughly mixed. (From Latour et al. 1981, courtesy ApJ.)

in the basic Navier Stokes equations. At that time, it was out of question to treat the problem in full three dimensions; to capture nevertheless some of the inherent threedimensionality, their idea was to project the temperature field on a set of horizontal functions $f_{k}(x, y)$, such that

$$
T(x, y, z, t)=\bar{T}(z, t)+\sum_{k} f_{k}(x, y) T_{k}(z, t)
$$

and likewise for the vertical velocity. The horizontal functions are chosen to be orthogonal and periodic in space, thus obeying the harmonic equation

$$
\nabla^{2} f_{k}(x, y)=-a_{k}^{2} f_{k}(x, y)
$$

with $a_{k}$ being the modulus of the wavenumber. Their products, which intervene in the non-linear terms of the governing equations, are characterized by the coupling constants $C^{k l m}=\frac{1}{2}<f_{k} f_{l} f_{m}>$. Le problem reduces then to a set of coupled differential equations in $t$ and $z$, whose solutions are the amplitude functions $T_{k}(z, t)$ and the mean temperature profile $\bar{T}(z, t)$.

Ed, Douglas and Juri had applied this method, the so-called modal approach, to laboratory convection, in the Boussinesq approximation which treats the fluid as if it were almost incompressible, and they had successfully resolved the thin boundary layers that control the heat flux (Gough et al. 1975; Toomre et al. 1977) - a numerical challenge. Now Douglas was leaving for Cambridge, and it was my turn to join the team, which we later nick-named "The Convective Collective". We were hosted by the Goddard Institute for Space Studies, in New York City. Sponsored by NASA, this Institute was equipped with the most powerful computer of that time, an IBM 360/95. Only two of these had been built, both for NASA, and IBM was proud to announce that they were able to perform "over 330 millions of 14 digit multiplications in one minute". This means that these super-computers were 100 times slower than a current laptop, or $10^{8}$ slower than 
today's petaflop computers. Ever since these New York years, Juri has managed to surf on the wave of the most powerful computers of their time. $\dagger$

We were granted a generous access to this computer by Robert Jastrow, the director of GISS. Our goal was to apply the modal approach to the convection zone of a star, and for the first attempt we chose an A-type star, which possesses two superposed unstable regions, due respectively to the ionization of hydrogen and helium. This problem looked easier to handle than that of the solar convection zone, because the density contrast is less extreme in such a star, and convection carries there a modest fraction of the heat flux. To filter out the acoustic waves, whose inclusion would have required much higher computer resources, we adopted the anelastic approximation, which had just been discussed by Douglas Gough (Gough 1969). Since we could not afford more than one or two horizontal wavenumbers in our modal expansions, the solutions were developing somewhat artificial internal boundary layers, in an attempt to build up an inertial cascade. We spent many nights watching the solutions on the screen of the IBM 2250 monitor, interacting with the light-pen to adjust the spatial resolution to the thinness of these boundary layers. Quite independently from the initial conditions, our solutions would settle in a stationary flow pattern, a far cry certainly from the turbulent regime that occurs in a real star.

Nevertheless, we had faith in our main result, namely that the convective motions overshoot the unstable regions by a substantial amount, sufficient to link the two unstable zones of an A-type star (Fig. 2) and thus to establish a single mixed subsurface region. This was later confirmed by more realistic simulations; it has observable consequences on the surface composition of such stars, which had not been explained until then. Our first results were published in two papers of the same issue of ApJ (Latour et al. 1976; Toomre et al. 1976), completed by a third paper in 1981 (Latour et al. 1981). In the meantime my student Jean Latour had joined our team, and even after our departure from New York, we would come back regularly during several years to pursue our computations at GISS. (Juri's parents, who were living on Long Island, kindly offered us to stay at their home on Long Island.)

In the summer of 1976, I organized with Ed Spiegel an IAU colloquium on "Problems of Stellar Convection", at the Nice observatory. It was attended by most people working in the field, among them the great pioneers Ludwig Biermann, Erika Böhm-Vitense and Paul Ledoux. Some promising young scientists were present too, such as Åke Nordlund who presented us a clever "thumbnail movie" of two-dimensional convection. There we saw also the first 3-dimensional simulation of compressible convection over multiple scaleheights, which had just been obtained by Eric Graham on the GISS computer (Graham 1977). And Fritz Busse showed us how rotation may interact with convection.

Clearly the time had come for more serious simulations than our crude modal solutions, and the computers had grown ready for the task.

\section{A serious improvement: convection in two dimensions}

In the fall of 1971 Juri had moved to the Joint Institute for Laboratory Astrophysics in Boulder to take a professorship at the University of Colorado. Rapidly he built up around him a team of students and postdocs dedicated to computational astrophysics. He first tackled the two-dimensional modeling of stratified convection, where the horizontal scale of the convective flows was no longer imposed as in the modal approach, but resulted

$\dagger$ This was also the computer on which Juri ran the simulations of the famous paper with his brother Alar on "Galactic Bridges and Tails" (Toomre \& Toomre 1972). 


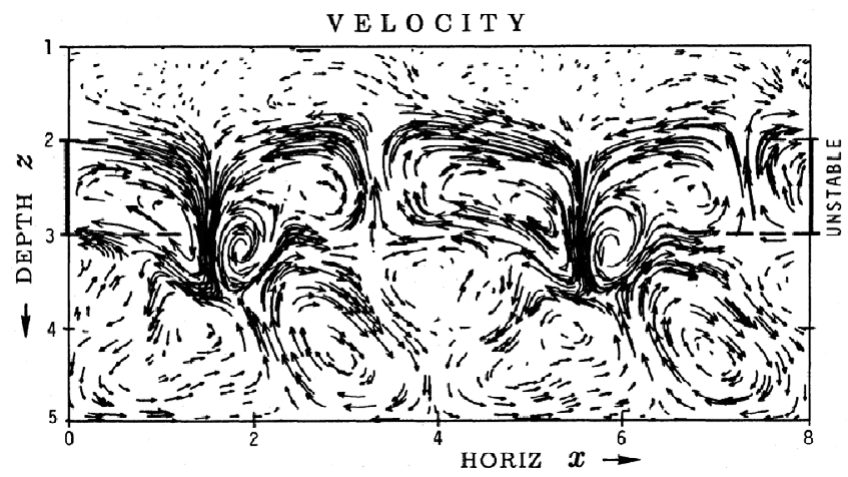

Figure 3. Two-dimensional simulation of stratified convection; the temperature contrast measures 5 between top and bottom of the computational domain. The unstable layer is located between two stable regions; its depth is taken as unit length. The downdrafts are much stronger than the upwellings, and they occupy less horizontal space. Note the deep penetration below into the stable region. (From Hurlburt et al. 1986, courtesy $A p J$ )

of the non-linear interactions of the problem. Moreover, the solutions are then no longer stationary, but display strong time dependence.

A snapshot of such a simulation is shown in Fig. 3, where an unstable layer is embedded between two stable regions (Hurlburt, Toomre \& Massaguer 1986). The striking property of the convective flows is that they do not remain confined in the unstable region, but that they dig deep into the stable region below, emitting gravity waves. This penetration is due mainly to strong downdrafts, which occupy a rather small fraction of the area, as required by the conservation of mass. The depth of penetration depends on the strength of the stable stratification, but also quite sensitively on the Peclet number, which measures the ratio between the advective and diffusive transports. This behavior was later confirmed by three-dimensional simulations, where the down-flows take the form of plumes (Brummell, Clune \& Toomre 2002). However, the exact penetration depth is still in debate, because the Péclet number which characterizes the base of the solar convection zone, $\approx 10^{6}$, remains out of reach of the numerical simulations, even with present-day supercomputers.

Juri was much aware of the need to validate the numerical simulations, because of their inherent limitations, through observations or laboratory experiments. Quite naturally he became extremely interested in the five minutes oscillations that had been observed on the Sun (Deubner 1975); it was clear that they could be used to probe the solar interior. His first paper on the subject was published in 1981 with Frank Hill and Larry November (Hill et al. 1981); it was followed by many others until now, in collaboration with most scientists working in the field. At the same time Juri engaged in active lobbying for helioseismology, both space-borne and ground based. All these efforts culminated in 1990 in a six months session he organized with Douglas Gough at the Institute for Theoretical Physics in Santa Barbara. All those who were working in the subject gathered there to spark off a new era in solar physics - it is worth browsing through the proceedings (Gough \& Toomre 1990). It is there that I learned that the transition from differential rotation in the convection zone to quasi uniform rotation in the radiative interior occurs in a surprisingly thin layer, which we later called the tachocline, when I proposed with Ed Spiegel its first, purely hydrodynamic model (Spiegel \& Zahn 1992). It is there also that Juri incited me to have a closer look on how the convective motions penetrate into a stable region. I guess that most participants benefited likewise from this stimulating meeting. And Juri and Douglas reached their goal: they obtained that the space mission SoHO (Solar \& Heliospheric Observatory), a joint project between ESA and NASA, be 

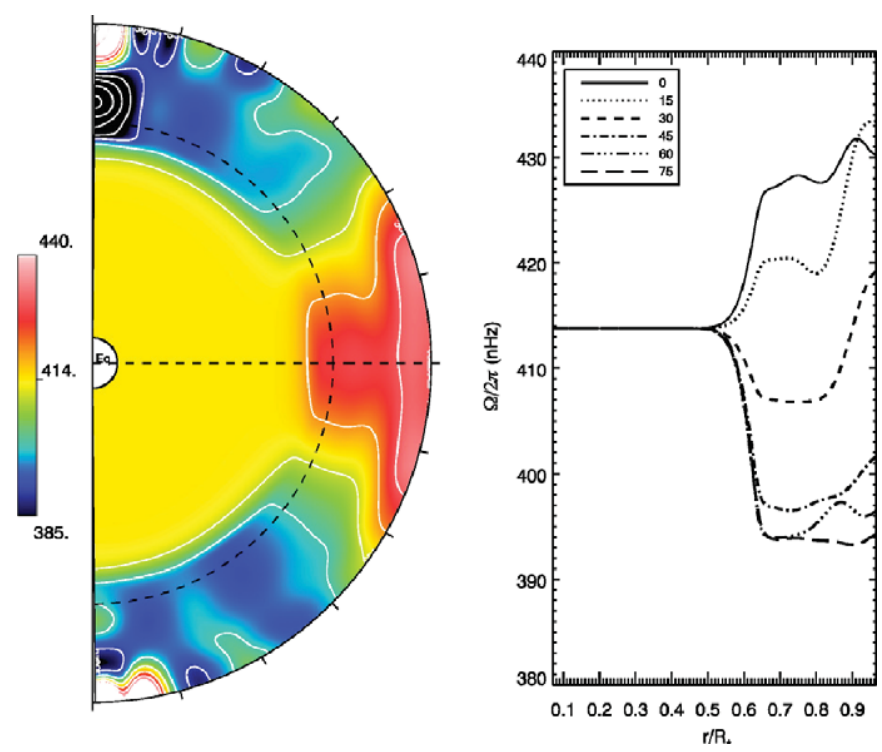

Figure 4. The solar interior rotation according to the ASH code (taking temporal and azimuthal averages). The computational domain encompasses $r / R_{\odot}=0.07$ to 0.97 , the numerical resolution is $N_{r} \times N_{\theta} \times N_{\varphi}=770 \times 256 \times 512$. On the right panel radial cuts at selected latitudes (after averaging the north and south hemispheres) reveal the strong shear in the tachocline naturally realized in the model. (From Brun et al. 2011, courtesy ApJ)

equipped with the instrument MDI (Michelson Doppler Imager), which was especially designed for high resolution helioseismology, while the network GONG was deployed with six sites covering the whole Earth, to ensure continuous monitoring of the Sun. These programs were extremely successful, and they gave a tremendous impetus to solar research.

For the same purpose of validating computer simulations, Juri engaged with John Hart and a few other colleagues in a microgravity experiment put on board of Spacelab 3 which was launched in May 1985 (Hart, Toomre et al. 1986). Thermal convection was triggered in a rotating, differentially heated hemispherical shell, with a radial buoyancy force created by an alternating electrostatic field. Excellent agreement was found between the convective patterns observed in the experiment and those obtained by Gary Glatzmaier with a three-dimensional numerical code.

\section{Convection on a global scale, in three dimensions}

It was Glatzmaier's code, written while he was preparing his $\mathrm{PhD}$ under the supervision of Peter Gilman, which represented the first serious attempt to model a whole convection zone in spherical geometry. Juri saw that this was the way to proceed, and with Mark Miesch and Tom Clune they redesigned Gary's code "to take full advantage of the higher resolution possible on currently available parallel super-computing platforms", as they announced at the AAS meeting in June 1996. Since then the code kept being adapted to the latest breed of super-computers, a task involving a growing number of collaborators that Juri was accreting. In this code, which is described in Clune et al. (1999), the acoustic waves are again filtered out by the anelastic approximation, and the horizontal surfaces are mapped by spherical harmonics while the vertical profiles are projected on Chebyshev polynomials. Hence the acronym ASH, for Anelastic Spherical Harmonics. 


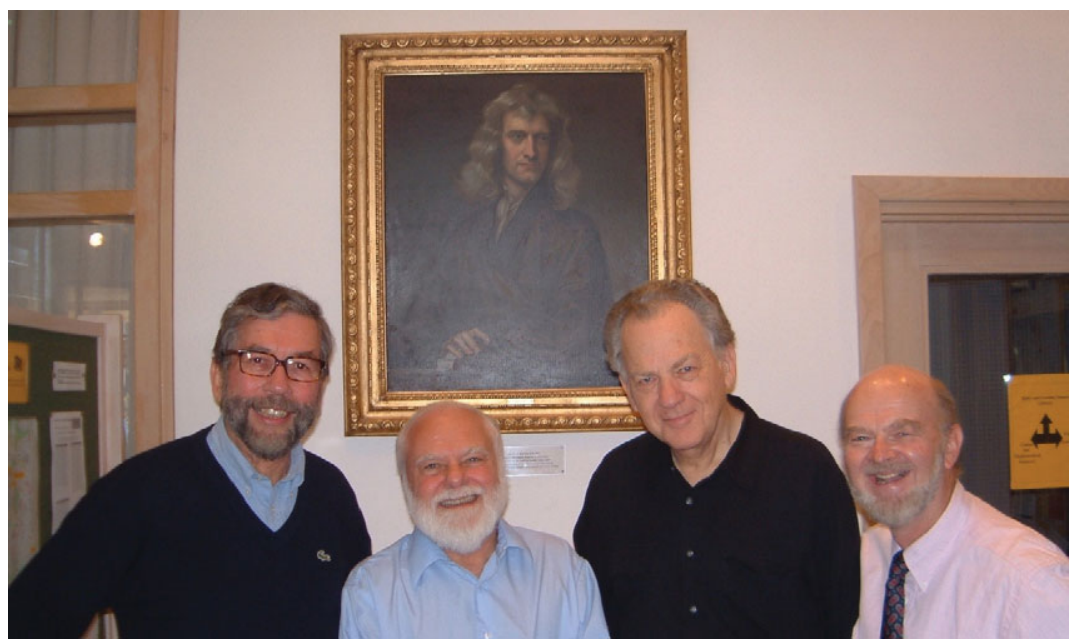

Figure 5. The Convective Collective reunited in 2004 at the Isaac Newton Institute in Cambridge, for a meeting on the solar tachocline (picture by A.S. Brun).

ASH succeeded where earlier calculations had failed: at last the rotation profile of the convection zone was compatible with that determined through helioseismology: the equator rotated faster than the poles and the differential rotation depended little on depth. Moreover, the cause of the equatorial acceleration was now identified: it is due to the transport of angular momentum from pole to equator through the Reynolds stresses (Brun \& Toomre 2002). The code has been applied since to various other problems, such as the young (thus fast rotating) suns and core convection in massive stars. The original hydrodynamic version has been extended to include the magnetic field (Brun, Miesch \& Toomre 2004), a significant improvement which enables it to treat the dynamo problem and to predict the evolution of a fossil magnetic field.

However one weakness of most of these simulations is that they require boundary conditions to be applied on the computational domain, which for numerical reasons cannot extend to the surface and is often confined to the convectively unstable region. It was found that the results are rather sensitive to a pole-equator variation of the temperature imposed at the lower boundary (Miesch, Brun \& Toomre 2006). This called clearly for including the tachocline in the computational domain, and the latest simulations reach even much deeper into the radiative core. Figure 4 displays such a state of the art calculation, where the tachocline is achieved without any artefact (Brun, Miesch \& Toomre 2011).

For completeness, I should mention that Juri participated also in many simulations performed in a cartesian box (cf. Cattaneo et al. 1991; Brummell, Clune \& Toomre 2002), which can be applied to specific problems or specific regions of a convection zone: subsurface layers, the lower boundary where penetration occurs. This was the path chosen by most other teams, with some putting their emphasis on a realistic treatment of radiative transfer, in order to predict the solar granulation pattern or the profile of spectral lines (cf. Stein \& Nordlund 1989; Asplund et al. 2000). But to me Juri remains above all the master of global simulation.

The tachocline is still arousing much interest. It certainly plays a key role in the solar dynamo, by shearing the poloidal field, pumped down from above, into toroidal field (the omega-mechanism), as it has been demonstrated through 3-D simulations (Browning et al. 2006). But it is still not clear why that layer is so thin. To confront the 
various explanations that have been elaborated, a meeting was organized in 2004 by David Hughes, Bob Rosner and Nigel Weiss, at the Isaac Newton Institute in Cambridge. For the first time in thirty five years, the four members of the Convective Collective were reunited. In the meanwhile, they had drifted their way. Ed became fascinated in dynamical systems and in more or less strange attractors. Douglas became the leading figure in theorizing helioseismology - and now asteroseismology. I turned to rotational mixing in stellar radiation zones. Only Juri stayed faithful to his early engagement: to provide the best representation of stellar convection with the most powerful computers. And his achievements are truly impressive, as we will again be reminded during this symposium, a tribute that his students and friends dedicate to him for his 70th birthday.

\section{References}

Asplund, M., Nordlund, A. A., Trampedach, R., Allende Preto, C., \& Stein, R. F. 2000, A\&̊A, 359,729

Biermann, L. 1938 AN, 264, 361

Browning, M., Miesch, M. S., Brun, A. S., \& Toomre, J. 2006 ApJ, 648, 157

Brummell, N. H., Clune, T. L., \& Toomre, J. 2002 ApJ, 570, 825

Brun, A. S., Miesch, M. S., \& Toomre, J. 2004 ApJ, 614, 1073

Brun, A. S., Miesch, M. S., \& Toomre, J. 2011 ApJ (submitted)

Brun, A. S. \& Toomre, J. 2002 ApJ, 570, 865

Canuto, V. M. 1992 ApJ, 392, 218

Cattaneo, F., Brummell, N. H., Toomre, J., Malagoli, A., \& Hurlburt, N. E. 1991, ApJ, 370, 282

Clune, T. L., Elliott, J. R., Glatzmaier, G. A., Miesch, M. S., \& Toomre, J. 1999 Parallel Comput., 25, 361

Cowling, T. G. $1936 A N, 258,133$

Deubner, F. L. 1965, A\&A, 44, 371

Gough, D. O. 1969 J. Atmospheric Sciences, 26, 448

Gough, D. O., Spiegel, E.A., \& Toomre, J. 1975 J. Fluid Mech., 68, 695

Gough, D. O. \& Toomre, J. (ed.) 1991 Helioseismology - probing the interior of a star; LNP vol. 388

Graham, E. 1977, Problems of Stellar Convection, IAU Coll. 38 (ed. E.A. Spiegel \& J.-P. Zahn), p. 151

Hart, J. E., Toomre, J., Deane, A. E., Hurlburt, N. E., Glatzmaier, G. A., Fichtl, G. H., Leslie, F., Fowlis, W. W., \& Gilman, P. A. 1986 Science, 243, 61

Hill, F., Toomre, J., \& November, L. J. 1981 BAAS, 13, 860

Hurlburt, N. E., Toomre, J., \& Massaguer, J.-M. 1986 ApJ, 311, 563

Latour, J., Spiegel, E. A., Toomre, J., \& Zahn, J.-P. 1976 ApJ, 207, 233

Latour, J., Toomre, J., \& Zahn, J.-P. 1981 ApJ, 248, 1081

Miesch, M. S., Brun, A. S., \& Toomre, J. 2006 ApJ, 641, 618

Miesch, M. S., Clune, T. C., Toomre, J., \& Glatzmaier, G. A. 1996, BAAS, 28, 936

Spiegel, E. A. \& Zahn, J.-P. 1992, A\& A, 265, 106

Stein, R. F. \& Nordlund, A. 1989 ApJ, 342, L95

Toomre, J., Gough, D.O., \& Spiegel, E.A., 1977 J. Fluid Mech., 125, 99

Toomre, J., Zahn, J.-P., Latour, J., \& Spiegel, E. A., 1976 ApJ, 207, 545

Unsöld, A. $1930 Z f A, 1,138$

Vitense, E. 1953 ZfA, 32, 135

Xiong, D. R. $1985 A \& 3 A, 150,133$ 